\title{
A Comprehensive Assessment of the Role of miRNAs as Biomarkers in Gastroenteropancreatic Neuroendocrine Tumors
}

\author{
Anna Malczewska ${ }^{a}$ Mark Kidd $^{\mathrm{b}}$ Somer Matar ${ }^{\mathrm{b}}$ Beata Kos-Kudla ${ }^{\mathrm{a}}$ \\ Irvin M. Modlin ${ }^{c}$ \\ ${ }^{a}$ Department of Endocrinology and Neuroendocrine Tumors, Medical University of Silesia, Katowice, Poland; \\ ${ }^{b}$ Wren Laboratories, Branford, CT, USA; ' $Y$ ale University School of Medicine, New Haven, CT, USA
}

\section{Keywords}

Biomarker · miRNA · Neuroendocrine neoplasia .

Neuroendocrine tumor

\begin{abstract}
Background/Aims: A key issue in neuroendocrine neoplasia management is the identification of blood signatures that specifically define the activity of a cancer or local tumor microenvironment. MicroRNAs (miRNAs) may represent such a candidate. To evaluate their clinical utility as biomarkers in gastroenteropancreatic neuroendocrine tumors (GEP-NETs), we assessed their expression in tissue and blood. Methods: A systematic review of PubMed was undertaken to identify studies investigating miRNAs in GEP-NETs and their utility as blood or tissue biomarkers. Results: Twenty-two studies using a range of methodologies with different normalization protocols were identified: tumor - gastric NET type 1 ( $n=1$ study: MiR-222, regulates $p 27$ KIP1), pancreatic $(n=6$ : MiR-21 [inflammatory marker, oncogene] and MiR-144 $\left[\mathrm{PI}_{3} \mathrm{~K} / \mathrm{AKT}\right.$ signaling], both up- and downregulated depending on the method), small intestinal ( $n=7$ : no consistent signature), and colorectal ( $n=3$ : no consistent signature); blood - gastric NET type 1 ( $n=1:$ MiR-222), pancreatic $(n=3:$ MiR-21), and small intestinal ( $n=3$ : no consistent signature). The studies
\end{abstract}

\section{KARGER}

(c) 2018 S. Karger AG, Basel

E-Mail karger@karger.com

www.karger.com/nen all included heterogeneous cohorts, were insufficiently powered, and utilized different methodologies, and ageand gender-matched controls were not used. Different miRNA isolation methods and detection protocols resulted in inconsistent expression comparing tumor and blood. A scientific discrepancy was the downregulated expression of some circulating candidates compared to tissue levels, suggesting methodological issues or physiological responses to the tumor. Both are of concern in defining the biometrics of a marker. Conclusions: A potential biomarker for GEP-NETs included MiR-21 (small bowel and pancreas), but this epithelial tumor marker requires prospective validation. Overall, significant scientific investigation remains to identify and demonstrate neuroendocrine specificity and to validate candidate miRNA biomarkers.

(c) 2018 S. Karger AG, Basel

\section{Introduction}

A key issue in the management of neoplasia, including neuroendocrine tumors (NETs), is the identification, in peripheral blood, of signals or signatures that specifically define the activity of the cancer or local tumor microenvironment [1]. The concept is captured within the terms 
"biomarker" and, more recently, "liquid biopsy." Numerous classes of analyte candidates have been identified and are under investigation. While biopsy and tissue-based markers are of considerable use, the real-time clinical utility of effective tools cannot depend upon multiple invasive procedures. Hence, sampling of biofluids (e.g., blood, urine, or saliva) with a view to the development of liquid biopsies has become an area of considerable medical and scientific interest.

Initially, circulating proteins were measured via enzyme-linked immunosorbent assay (e.g., carcinoembryonic antigen, prostate-specific antigen, chromogranin A, etc.), but their limitations in terms of dimensionality, coupled with a modest specificity, diminished the enthusiasm with regard to their clinical utility [2]. A wide variety of surrogates for delineating clinical neoplastic status - liquid biopsies - have been proposed and investigated with varying degrees of effectiveness $[1,3,4]$. These include circulating tumor cells, tumor-derived DNA in the circulation, mRNAs, and recently microRNAs (miRNAs). All have been proposed to provide information pertinent to defining the evolution of cancer in a particular individual, and each appears to provide information that might be of considerable utility. As yet, however, no molecular signature has accurately captured the behavior of an individual neoplasm such that definitive clinical management decisions can be implemented.

Recent biomarker candidates are miRNAs, which comprise a family of short ( $<30$ nucleotides) noncoding RNAs purported to regulate a diverse array of biological processes including carcinogenesis $[5,6]$. Of particular note is that in neoplastic cells, miRNAs have been identified to be substantially dysregulated [7]. This may reflect the involvement of multiple mechanisms including, but not limited to, amplification or deletion of miRNA genes, abnormal transcriptional control, epigenetic modifications, or defects in the miRNA biogenetic machinery [8]. miRNAs have multiple targets, and their putative role in tumorigenesis or the advance of the neoplastic processes reflects their influence on a range of targets involved in regulating specific pathways [9]. Once abnormal miRNA expression (gain or loss) occurs, cells are anticipated to acquire the capability to develop independent proliferative signaling, elude growth suppressor regulation, ameliorate cell death, induce angiogenesis, and concomitantly activate invasion and metastasis [8].

To explore the clinical utility of miRNAs as a viable biomarker, information regarding measurement techniques, tumor-specific signatures, and miRNA clusters that define particular biological processes is required. Genome-wide profiling has established that miRNA expression signatures are related to the tumor type, tumor grade, and clinical outcomes. There is thus a high likelihood that miRNAs could be candidate diagnostic or prognostic biomarkers or even therapeutic targets [10-16].

The goal of this review is to assess the role of miRNAs in neuroendocrine neoplasia with a view to assessing the likelihood that measurement of a miRNA biomarker signature will be of clinical utility in assessing diagnosis, prognosis, or response to treatment. A systematic review of the literature in PubMed was undertaken (Fig. 1). The search criteria included "(miRNA OR microRNA) AND neuroendocrine NOT (melanoma OR pheochromocytoma OR paraganglioma)" and all studies reporting dysregulated miRNA expression in gastroenteropancreatic NETs (GEP-NETs; blood or tissue) were identified. To assure that all relevant studies were included, the references of the manuscripts initially identified were also evaluated against the primary inclusion criteria. One additional investigation was identified. Therefore, 22 articles were included in the final analysis.

\section{Biology of miRNAs}

miRNAs are short ( 22 nucleotides in length) noncoding RNA molecules that regulate gene expression negatively at a posttranscriptional level [17]. Since the discovery of lin-4 in 1993 in Caenorhabditis elegans [18], 2,588 human miRNAs have been identified, and their genome location, sequence, and transcript annotation are available (miRBase v.21) [19].

miRNA genes are initially transcribed by nuclear RNA polymerase II into long primary miRNA transcripts (primiRNAs). These are characterized by a $5^{\prime}$ cap structure (7mGpppG) as well as a $3^{\prime}$ end poly(A) tail. miRNA genes can be either transcribed as monocistronic or polycistronic (e.g., MiR-17-92-1 cluster) outputs. Transcriptional regulation is controlled by host tissue genes.

Mature miRNAs are synthesized from precursors with a hairpin structure by ribonucleases (nuclear RNase III DROSHA). This complex generates 60-100 nucleotide pre-miRNA products, which fold into stable secondary stem-loop structures. Pre-miRNAs are exported into the cytoplasm by the Ran-GTP-dependent transporter exportin 5, where they are processed by a second RNase III enzyme, DICER, which excises the terminal loop, releasing transitory double-stranded RNA duplexes (Fig. 2) [20]. Once processed and embedded into the Argonaute 
INCLUSION CRITERIA:

GEP NET studies that reported 1) miRNA dysregulated expression and/or 2) evaluated miRNAs as blood or tissue biomarkers

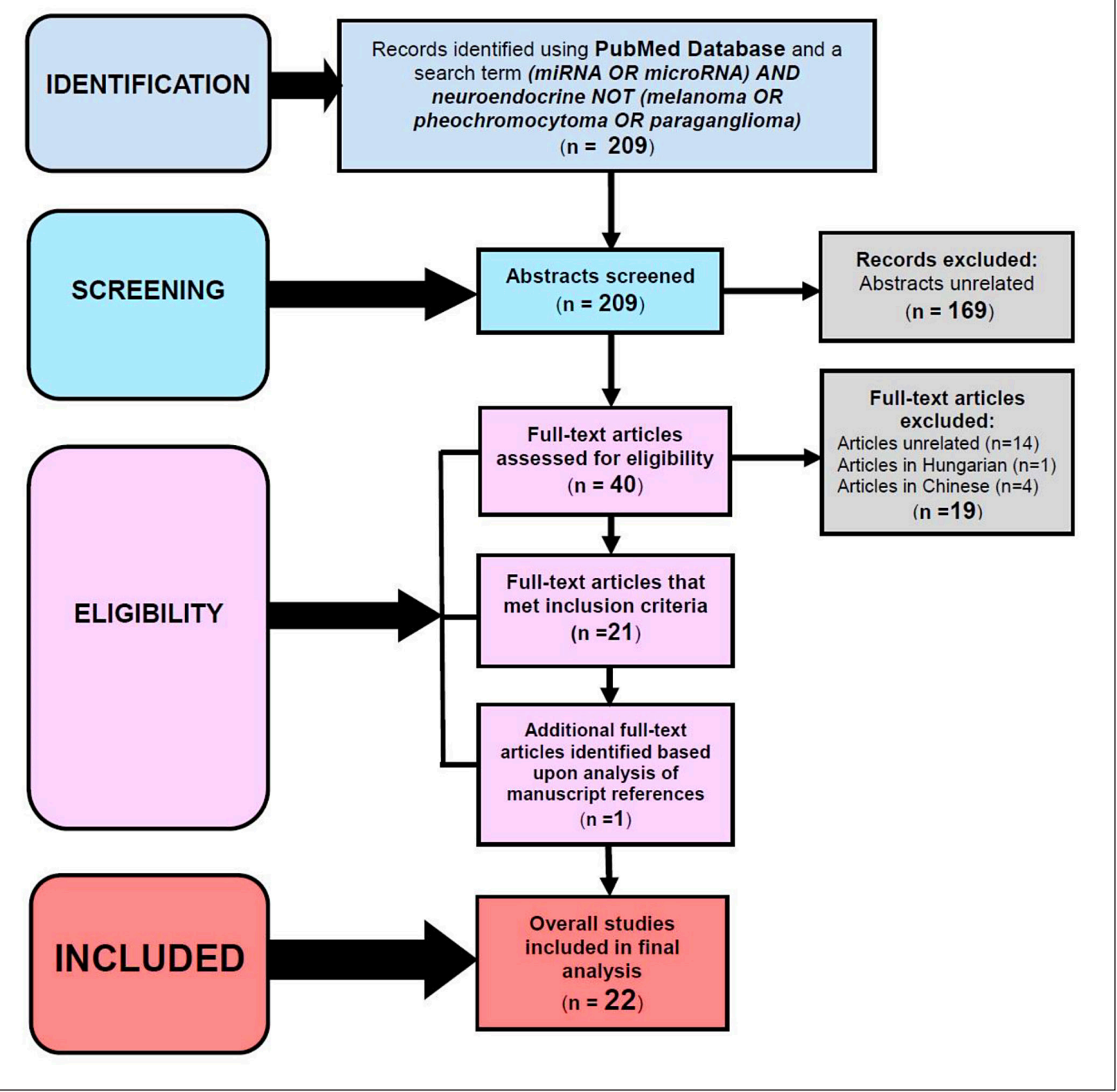

Fig. 1. PRISMA flow diagram of the systematic review performed. GEP NET, gastroenteropancreatic neuroendocrine tumor.

protein of the miRNA-induced silencing complex (RISC) [21], the miRNA seed region pairs with complementary sequences, located within $3^{\prime}$ untranslated regions $\left(3^{\prime}\right.$ UTR) [22], 5' UTR [23], or coding sequences [24] of the target mRNA. This silencing mechanism culminates in translational repression or mRNA cleavage [17]. The role of miRNAs in protein-encoding gene regulation is substantial: they are estimated to regulate $\sim 60 \%$ of all coding genes [25]. Thus, a single miRNA can target hundreds to thousands of mRNAs - and, conversely, a single mRNA may be regulated by multiple miRNAs [26]. Overall, therefore, miRNAs may be involved in the regulation of all quintessential cellular processes, including proliferation, differentiation, developmental timing, patterning of the nervous system, metabolism, signaling pathways, apoptosis, or hematopoiesis [17, 27-31]. 
Fig. 2. Schematic delineating miRNA nuclear production and delivery into the circulation. Mature miRNAs are synthesized from precursors (pri- and pre-miRNAs) by the ribonucleases Drosha and Dicer. Thereafter, they are embedded in the Argonaute protein of the miRNA-induced silencing complex (RISC), which is responsible for translational repression or mRNA cleavage following pairing with mRNA complementary sequences located within $3^{\prime}$ UTR, $5^{\prime}$ UTR, or coding sequences. The mechanism by which miRNA enters the circulation remains to be defined. Candidate mechanisms and potential pathways include leakage from damaged cells, or secretion into encapsulated microvesicles (e.g., oncosomes), into shedding vesicles such as exosomes, or into complexes (protein bodies) with different RNA-binding proteins (e.g., Argonaute-2 [Ago2], nucleophosmin [NPM1], or high-density lipoprotein).

\section{MiRNA COMPARTMENTS}

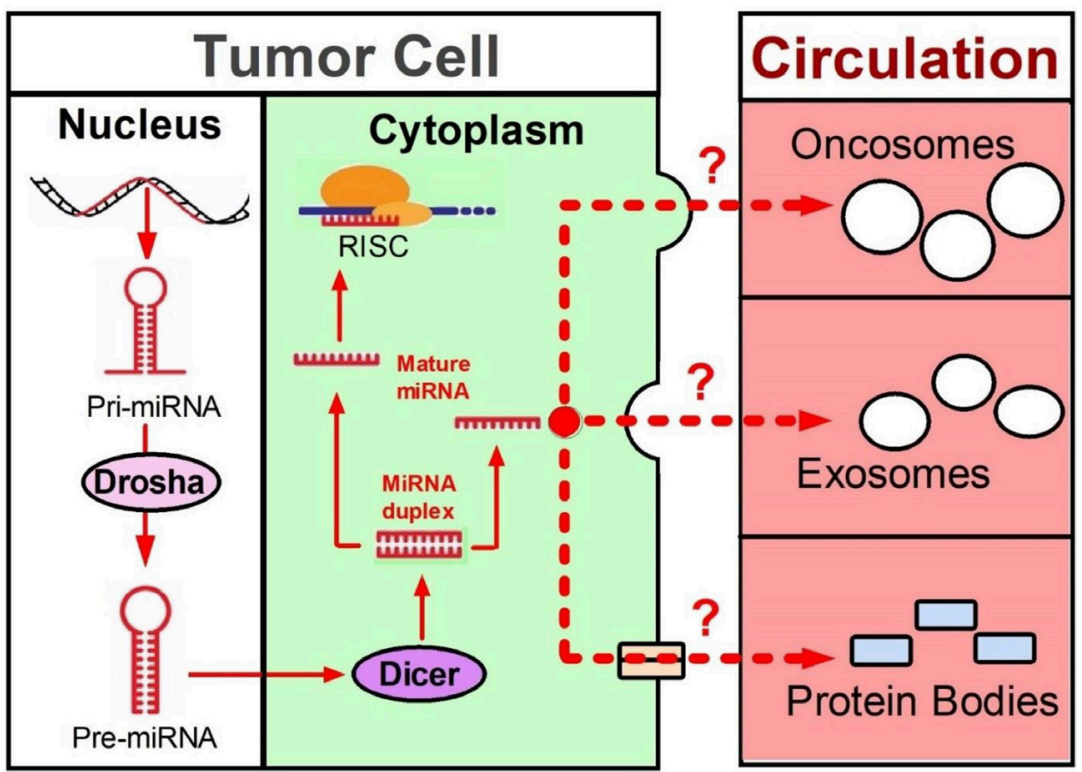

\section{miRNAs in Neoplasia}

In the last decade, the detection of miRNAs in body fluids [32-34] has initiated numerous investigations of circulating miRNA expression patterns in diverse pathological conditions. The data indicate a wide pattern of dysregulated circulating miRNA expression profiles in numerous disorders including cardiovascular, respiratory, renal, infectious, metabolic, neurodegenerative, and psychiatric diseases (Fig. 3) [35-38]. A predominant pattern of dysregulation has been identified in cancer [1016]. Based upon this relationship, the investigation of circulating miRNAs as novel cancer biomarkers has elicited considerable interest.

miRNAs are recognized as oncogenes and tumor suppressors $[5,6]$, and their aberrant expression has been linked to tumorigenesis and defined as a fundamental cancer hallmark $[8,39]$. Evidence of miRNA involvement in neoplasia was initially documented in 2002 after characterization of the frequently deleted chromosome 13q14 in chronic lymphocytic leukemia [40]. This encodes MiR$15 a$ and $-16-1$, which are expressed in the same polycistronic RNA [40]. They regulate $b c l-2$ expression, thus providing the genetic basis for BCL2 overexpression in chronic lymphocytic leukemia and the resultant loss of apoptosis. The underlying causes of aberrant miRNA gene expression are now known to be related to their location in cancer-associated genomic regions, or are due to epigenetic alterations (with altered transcription) or an aberrant miRNA processing machinery (with consequent up- and/or downregulation) [8].

Tissue profiling studies have identified tumor-specific miRNA profiles; accordingly, organ-specific, cancerassociated miRNA signatures have been detected [7]. To date, for each cancer type analyzed (and often stratified by stage), miRNA signatures have been identified that may be prognostic and/or predictive [41, 42]. Distinct miRNA panels of a primary and its metastases, in either clinical sets or preclinical models, have been reported [43]. Circulating miRNA expression has been proposed as a fingerprint to diagnose or prognose a specific cancer type [44]. Correlations between miRNA expression patterns and response to treatment have also been reported and may serve as predictors of either drug resistance or sensitivity to a particular therapy [45-47]. Moreover, miRNA expression profiles in metastasized, undifferentiated cancers or cancers of unknown primary origin are considered a potential guide to the identification of a primary tumor $[48,49]$. 


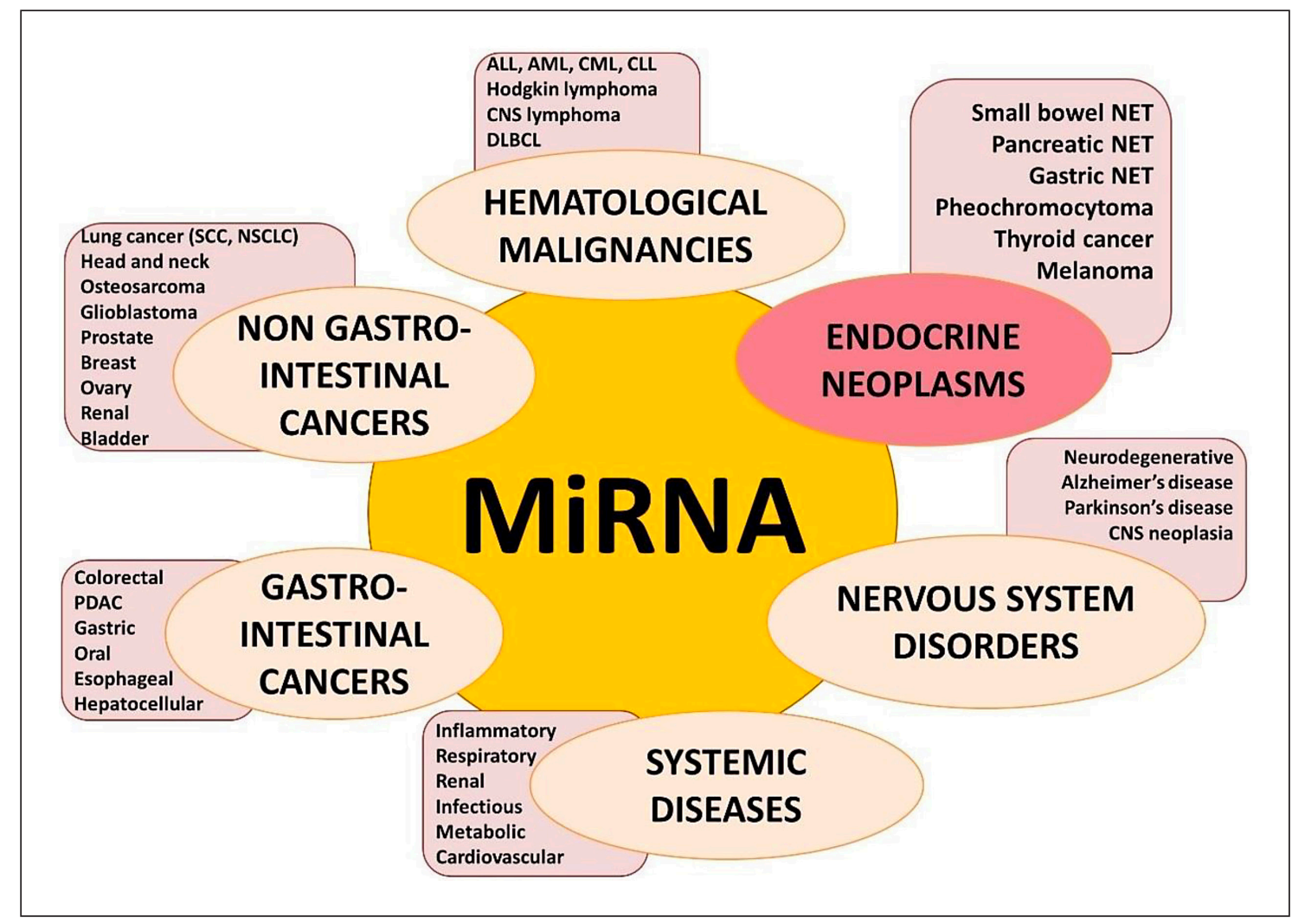

Fig. 3. The spectrum of diseases exhibiting dysregulated miRNAs. Diverse patterns of dysregulated circulating miRNA expression profiles have been identified in numerous pathological conditions including cardiovascular, respiratory, renal, infectious, metabolic, neurodegenerative, and neoplastic. Given that miRNA is regarded as a cancer hallmark, it is apparent from diverse investigations of neoplasia that miRNA dysregulation is ubiquitous. Such studies include lung, colorectal, breast, pancreatic, small intestinal, or pancreatic NET, thyroid cancer, melanoma, and pheochromocytoma among others. ALL, acute lymphoblastic leukemia; AML, acute myeloid leukemia; CML, chronic myeloid leukemia; CLL, chronic lymphocytic leukemia; DLBCL, diffuse large B-cell lymphoma; SCC, squamous cell carcinoma; NSCLC, non-small cell lung cancer; NET, neuroendocrine tumor; PDAC, pancreatic ductal adenocarcinoma.

\section{Tissue Expression of miRNAs in GEP-NETs}

In contrast to many other tumor types, little is known about the miRNA expression patterns of GEP-NETs.

\section{Gastric NET Type 1}

No tissue-based (global profiling) data have been published for tumors of the stomach, but $M i R-222$, which functions through downregulation of the cell cycle inhibitor $P 27^{K I P 1}$, has been identified in gastric NETs [50].

\section{Pancreatic NET}

Expression of the homologues MiR-103 and -107 (both involved in insulin sensitivity), along with loss of $M i R$ 155 , has been reported to discriminate sporadic pancreatic NETs (PNETs) $(n=40$; functioning [insulinoma; $n=$
12] and nonfunctioning [NF; $n=28$ ]; a total of 22 were well-differentiated NETs and 18 were invasive/metastasized NETs; no age or gender distribution provided) and acinar cell carcinomas $(n=4)$ from normal pancreatic islets $(n=12)$, suggesting these miRNAs may be involved in pancreatic tumorigenesis [51]. MiR-155 overexpression silences the proapoptotic tumor protein p53 inducible nuclear protein 1 (TP53INP1) in pancreatic ductal adenocarcinoma (PDAC), resulting in apoptosis evasion and uncontrolled growth. A separate panel of 10 miRNAs differentiated these 40 PNETs from the 4 pancreatic acinar cell carcinomas (and normal pancreas). These 10 miRNAs were proposed either to be associated with normal endocrine differentiation or as markers of endocrine tumorigenesis. Seven of the 10 were validated by a Northern blot (Table 1) [51]. 
Table 1. Overview of dysregulated miRNAs in GEP-NET tumor and blood compartments

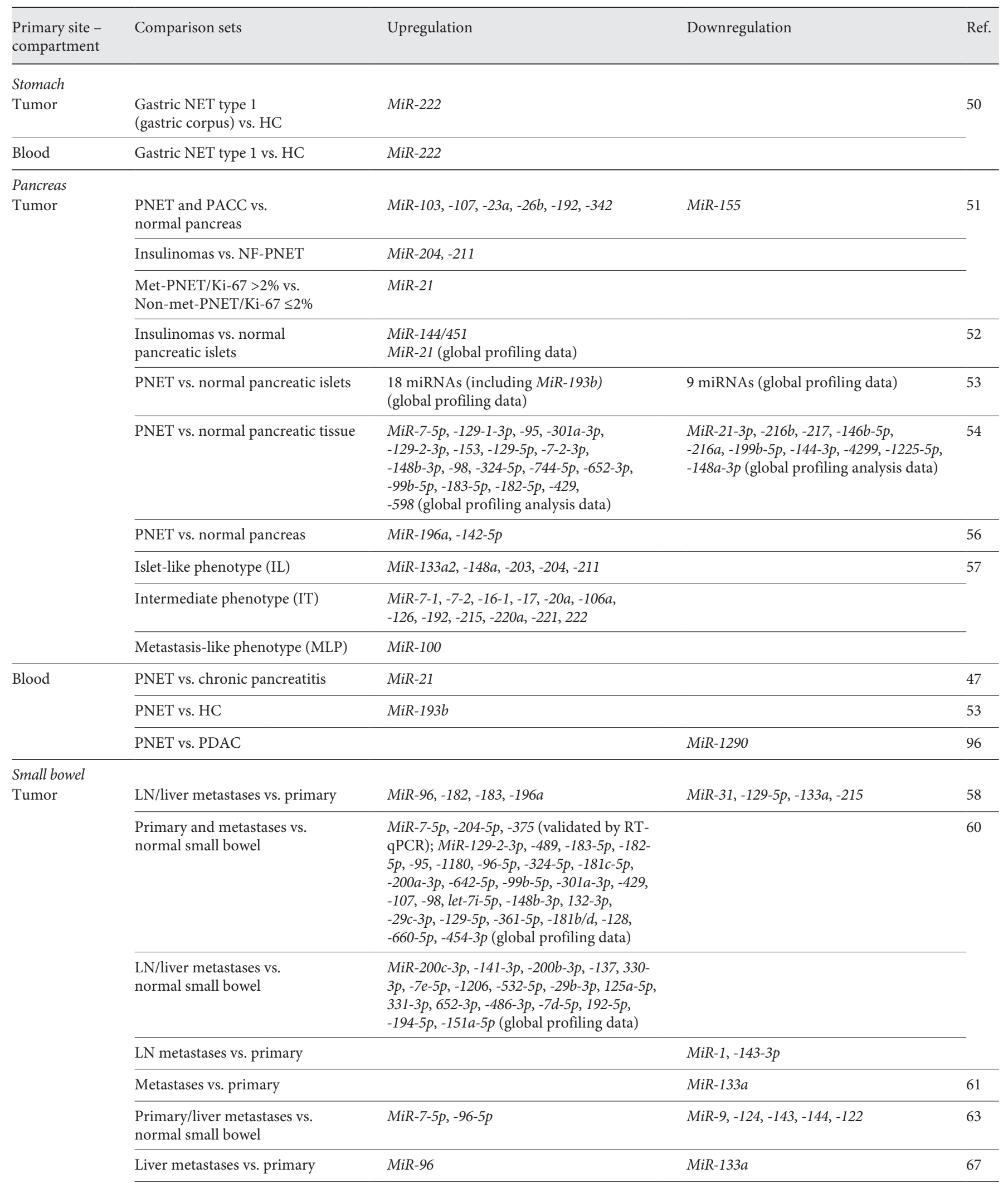


Table 1 (continued)

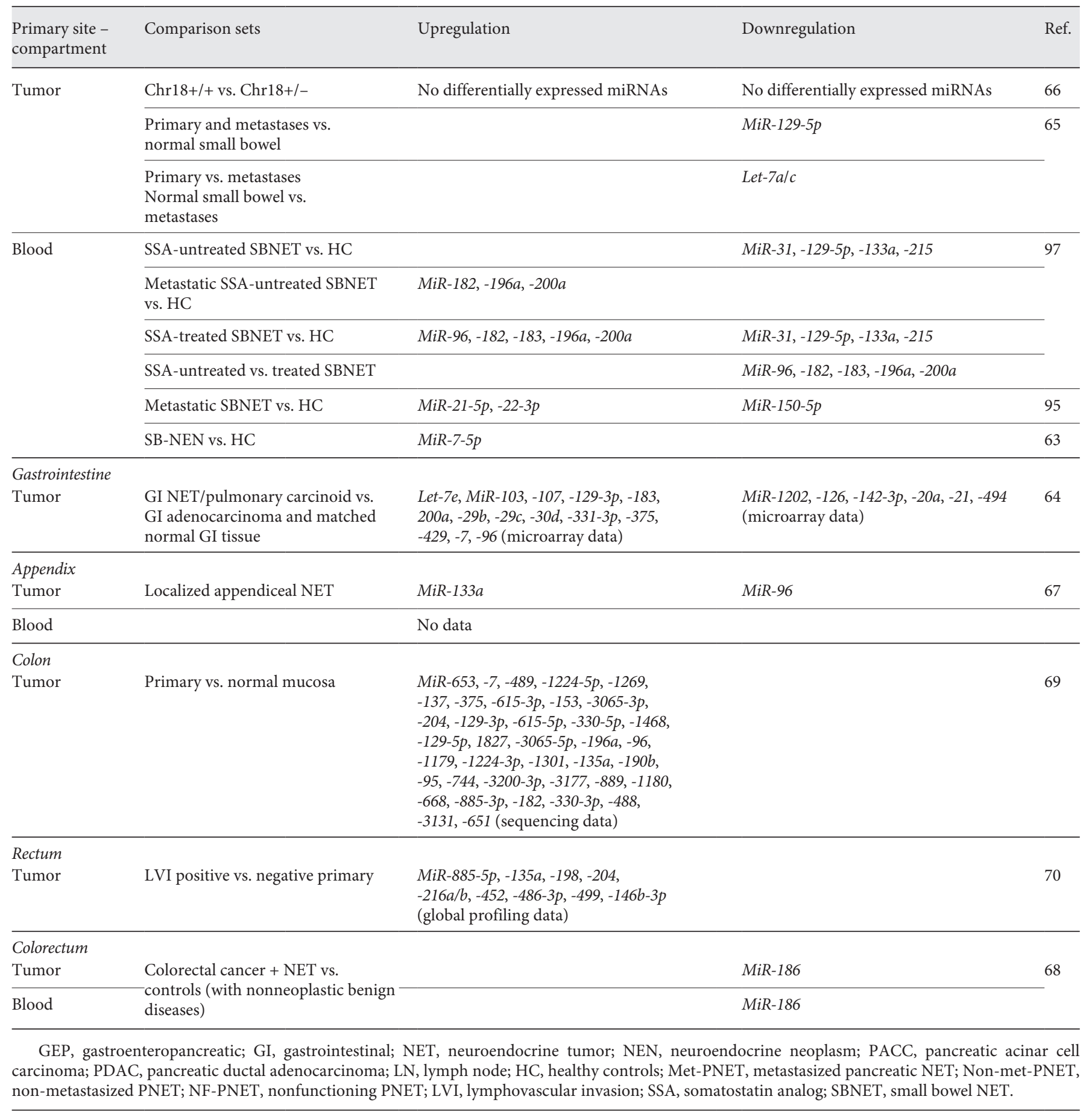


miRNA RT-qPCR array analysis of 4 insulinomas (no data provided in regard to age, gender, or histology) was compared to that of normal pancreatic islets $(n=4)$, which revealed 114 differentially expressed miRNAs. Amongst these, 28 miRNAs belonging to 3 miRNA families were localized in the epigenetically regulated imprinted chromosome 14q32 region. The most significantly differentially expressed miRNA cluster, $M i R$ 144/451 (oxidative stress tolerance, $\mathrm{PI}_{3} \mathrm{~K} / \mathrm{AKT}$ signaling), was validated by RT-qPCR in a separate group of 25 insulinomas and 8 normal pancreatic islet samples. This confirmed its overexpression in insulinomas. $M i R$ $144 / 451$ was also studied in murine pancreatic $\beta$-cells, where it induced cell proliferation. In the MIN6 cell line, MiR-144 targeted PTEN and activated the AKT pathway in pancreatic $\beta$-cells, while MiR-451 targeted the cell cycle regulator $P 19^{I N K 4 D}$ to promote pancreatic $\beta$-cell proliferation [52].

In the previously discussed study [51], MiR-204 (tumor suppressor, promoting apoptosis) was confirmed to be selectively expressed in insulinomas $(n=12$; age and gender not specified) compared to NF-PNETs, and to be correlated with insulin immunohistochemical expression. Amongst PNETs, the homologues MiR-203 and -211 (involved in suppression of tumor proliferation and migration) were upregulated in insulinomas. In the same study, overexpression of the cancer-ubiquitous MiR-21 was associated with both a higher proliferation index and the presence of liver metastases [51]. MiR-21 differentiated metastasized NF-PNETs/PNETs with Ki-67 >2\% from non-metastasized NF-PNETs/PNETs with Ki-67 $\leq 2 \%$, suggesting an association with proliferation. In contrast, a separate study on 37 PNETs (predominantly well differentiated; two-thirds with Ki- $67<2 \%$; no information in regard to functionality status; patient age $\sim 53$ years, $46 \%$ male) reported that expression of the adipocyte-specific MiR-642 but not of MiR-21 correlated with proliferation [53]. Moreover, only MiR-210 (regulated by hypoxia) correlated with metastatic disease [53]. The TNF-related apoptosis-inducing ligand (TRAIL) pathway-associated miRNA MiR-193b, linked to mesenchymal stem cell proliferation or silencing of embryonic stem cell renewal, was also overexpressed [53].

Twenty-eight differentially expressed miRNAs were identified [54] in transcriptomes from 6 Korean PNETs (Ki-67 and functional status unknown; 33\% with distant metastases; patient age $\sim 48$ years, $67 \%$ male) compared to 5 healthy pancreatic samples (not specified whether from exocrine pancreas or pancreatic islets [55]). Amongst the dysregulated miRNAs, 18 were upregulated and 10 downregulated, including MiR-21 and -144 (Table 1) [54]. In a separate study, 18 candidate miRNAs including $M i R-196 a$ (upregulated in primary tumors, regulator of HOX gene expression and AKT signaling) were identified to be differentially expressed in matched primaries and liver metastases $(n=2)$ [56]. In a validation sample of 37 PNETs (predominantly sporadic [92\%]; NF, 76\%; twothirds with Ki-67 <2\%; 84\% N0; patient age 57 years, $45 \%$ male), only MiR-196a was identified as prognostic. Tumors with elevated expression of this miRNA were associated with decreased disease-free and overall survival [56].

More recently, Hanahan and colleagues [57] identified three different genetic PNET subtypes with a spectrum of behavior and metastasis that did not correlate with the WHO classification system. In this study [57], miRNA profiles were leveraged with gene expression patterns to identify three different phenotypes of PNET: (1) islet-like (IL), (2) intermediate (IT), and (3) metastasis-like primary (MLP). Expression was associated with differences in genomic mutations, that is, the IT phenotype was not associated with $D A X X / A T R X$ mutations but with $M E N-1$ alterations, while the MLP phenotype exhibited DAXX/ $A T R X$ mutations and was both more metabolically active as well as likely to have evolved from a less well-differentiated progenitor cell. The IL phenotype was associated with upregulation of MiR-133a2, -148a, -203, -204, and -211 , and the IT phenotype with MiR-7-1, -7-2, -16-1, -17, $-20 a,-106 a,-126,-192,-215,-220 a,-221$, and -222 . The MLP phenotype was linked to $M i R-100$. The IT and MLP phenotypes overexpressed MiR-103 and -107. It is noteworthy that MiR-21, $-144,-196 a$, and -451 were not identified in this set.

\section{Small Intestinal/Bowel and Appendiceal NET}

In a study of 15 small bowel NETs (SBNETs; 5 primary tumors, all G1; 5 mesenteric metastases, $40 \%$ G1; 5 liver metastases, $60 \% \mathrm{G} 1$ ), global profiling identified 33 differentially regulated miRNAs in mesenteric metastases and 33 differentially expressed miRNAs in liver metastases versus primary tumors. Eighteen were upregulated. In a validation sample of 9 tumors $(67 \%$ G1, 67\% metastases), 4 miRNAs were upregulated in the primary tumor compared to metastases (MiR-31 [HDAC/CDK2 regulation], MiR-129-5 [HDAC-mediated cell death], MiR$133 a$ [cardiac specific], and MiR-215 [inhibitor of migration]). Separately, MiR-96 (proliferation and invasion), MiR-182 (BCL2 inhibitor), MiR-183 (inhibitor of cell invasion), and MiR-196a were upregulated in metastases versus the primary tumor (Table 1) [58].
$8 \quad$ Neuroendocrinology DOI: $10.1159 / 000487326$
Malczewska/Kidd/Matar/Kos-Kudla/ Modlin 
Based upon reports of MiR-196a dysregulation in SBNET [58] (and lung NET [59]), studies were undertaken in which MiR-196a was silenced in CNDT2.5 (midgut NET) and NCI-H727 (bronchial NET) cell lines to assess whether it was a negative regulator of its predicted target genes involved in multicellular organismal development (HOXA9 and HOXB7) or in the Wnt signaling pathway (LRP4 and RSPO2). All target genes and proteins, as well as 6 downstream $M i R-196$ genes, were significantly upregulated in the silenced NET cells, demonstrating no relationship between miRNA and target mRNAs. Additionally, the effect of MiR-196a silencing on cell proliferation was evaluated by the MTT assay, showing no significant regulation. Based on the study results, a potential regulatory effect of MiR-196a on cell growth control in these model cell lines was excluded [59].

Another SBNET global profiling analysis comprised two stages: (1) first global profiling of 15 SBNETs (mean patient age $\sim 68$ years, $47 \%$ female; $80 \%$ G1, $53 \%$ with liver metastases; formalin-fixed, paraffin-embedded [FFPE] samples: primary SBNET $[n=15]$; adjacent normal small bowel [NSB], $n=12$; matched lymph node metastases, $n=9$; normal lymph node, $n=2$; liver metastases, $n=2$ ) and (2) second profiling (fresh frozen samples: primary tumor, $n=13$; lymph node metastases, $n=15$; liver metastases, $n=13$; NSB, $n=2$ ). A comparative miRNA expression analysis of the primary SBNET and NSB samples from both profiling sets revealed 39 dysregulated miRNAs [60]. A comparison of the SBNETs and their metastases (lymph node and liver) with the normal tissues identified a 29-miRNA signature (Table 1) [60]. The 3 most upregulated miRNAs in SBNET (MiR-7-5p, -204-5p, and -375) were confirmed by RT-qPCR. MiR-7-5p inhibits cell proliferation/induces apoptosis, $M i R-204-5 p$ inhibits cell proliferation/ invasion, and $M i R-375$ is a pro-proliferative factor. Two miRNAs (MiR-1 [inhibitor of angiogenesis] and MiR143-3p [antiproliferative]) were significantly downregulated in lymph nodes $(n=24)$ and liver metastases $(n=$ 15) compared with primary tumors.

In a separate study, in SBNETs $(n=8)$ (all metastatic, 7 to the liver, 1 to a lymph node; no histology provided), $M i R-19 a+b$ (inhibitor of SMAD4/PTEN signaling) and $M i R-183$ and -488 (inhibit proliferation) were upregulated, while the MiR-133a, $-145,-146,-222$, and $-10 b$ (cardiac specific/involved in cancer development) were downregulated in metastases [61]. In an additional study of SBNETs $(n=15)$ (primary tumors; all patients with lymph node involvement, 9 with distant metastases; tumor grades not specified in this cohort), a miScript
miRNA PCR array analysis of 84 miRNAs was undertaken [62]. The data were normalized to SNORD95 and compared to a mix of 7 samples from normal whole small intestinal tissue. Twenty-six downregulated and 2 upregulated miRNAs were identified. The 5 most downregulated miRNAs (MiR-9, $-124,-143,-144$, and -122) and the 2 upregulated miRNAs (MiR-7-5p and -96-5p) were then further validated in an additional 15 tumor samples (6 primary tumors and 9 liver metastases). The liver metastases showed a dysregulation pattern similar to that of the primary tumors. MiR-7-5p was significantly upregulated in all SBNET samples, irrespective of the tumor size or stage, and was therefore selected for further testing in serum (see section miRNAs as a Circulating Marker in GEP-NETs below). Suggested potential targets and regulators of MiR-7 comprised Yin Yang 1 (YY1), NF- $\mathrm{kB} /$ RelA, and the proto-oncogene c-Myc [63], while MiR-7$5 p$ is a known tumor suppressor in many cancer types, inhibiting growth and invasion by suppressing expression and activity of the EGFR signaling pathway, or NF$\kappa \mathrm{B} /$ RelA signaling (e.g., in melanoma). Overexpression of $M i R-7-5 p$ has been found to inhibit cell proliferation and induce apoptosis by targeting REG $\gamma$ in vitro and in vivo in breast cancer cells [62].

A miRNA microarray analysis of primary gastrointestinal (GI) NETs $(n=28)$ ( $86 \%$ G1, $14 \%$ G2; gastric, $n=7$; duodenal, $n=9$; rectal, $n=12 ; 50 \%$ metastasized) and pulmonary carcinoids ( $n=22 ; 82 \%$ typical, $18 \%$ atypical, $18 \%$ metastasized) versus adenocarcinomas $(n=9)$ (pulmonary, $n=3$; gastric, $n=3$; rectal, $n=3 ; 67 \%$ metastasized) and normal tissue $(n=15)$ (collected from pulmonary $[n=6]$, gastric $[n=3]$, duodenal $[n=3]$, and rectal $[n=3]$ carcinoid cases) was undertaken in FFPE samples [64]. The majority of the NETs (48/50) formed one major cluster, whereas all adenocarcinomas, the normal tissue, and 2 atypical pulmonary carcinoids formed a separate cluster. The miRNAs differentially expressed $(n=20)$ between these clusters were upregulated miRNAs $(n=14)$ (Let-7e and MiR-103, -107, -129-3p, -183, -200a, -29b, $-29 c,-30 d,-331-3 p,-375,-429,-7$, and -96) and downregulated miRNAs $(n=6)(M i R-1202,-126,-142-3 p,-20 a$, -21 , and -494). The results suggest a common origin of GI and lung carcinoids. A comparison of the metastasized $(n=18)$ and localized NET cases $(n=29)$ did not, however, reveal any significant differences in miRNA expression [64].

A separate miRNA array profiling study examined 9 SBNET patients (G1: $n=5 ; \mathrm{G} 2: n=2 ; \mathrm{G} 3: n=1$ ), and it included 2 colonic samples [65]. A comparison of the primary tumor and metastases with NSB tissue revealed 48 
differentially expressed miRNAs out of 1,174 screened, of which 18 (including MiR-129-5p) were downregulated and 30 upregulated. A comparison of the metastases and normal tissue with the primaries revealed 145 dysregulated miRNAs (77 downregulated and 68 upregulated), whereas comparing the primary tumors with metastases revealed 163 differentially expressed miRNAs. Both comparisons showed downregulation of the let-7 family. To validate the results for MiR-129-5p and the let-7f/let-7 family, qPCR and in situ hybridization (ISH) was performed on a separate cohort. Only MiR-129-5p and let-7a and $c$ were confirmed by the qPCR analysis in microdissected tissue [65].

SBNETs are commonly $(\sim 66 \%)$ characterized by loss of chromosome 18 (Chr18), although the mechanistic relevance to disease progression has not been established [66]. An analysis of genetic, epigenetic, and gene/protein expression levels was undertaken in individuals with and those without loss of Chr18. Chr18-related miRNA ( $n=$ 27) expression in Chr18+/- $(n=10)$ and Chr18+/+ $(n=$ 10) SBNET samples was compared. Apart from the DCC (deleted in colorectal cancer) tumor suppressor, no significant genetic or epigenetic alterations, including those in Chr18-related miRNA expression, were identified. This suggests that loss of miRNA encoded on Chr18 is not of biological significance [66].

In a more recent study [67], RT-qPCR and ISH were performed to detect MiR-96 and -133a expression in FFPE samples from 51 primary GI neoplasms (mainly small intestinal, G1/G2, $n=50$; G3 with Ki-67 at 90\%, $n=1)$, matching lymph node metastases $(n=33)$ and liver metastases $(n=20)$. The RT-qPCR results for $M i R$ 96 expression were concordant with the ISH findings: a significantly higher expression was noted in the liver metastases. MiR-133a was downregulated in the metastases; ISH showed a similar trend, but this was not statistically significant.

Localized appendiceal carcinoids $(n=3)$ had low levels of MiR-96 and high levels of MiR-133a expression (qPCR). MiR-96 and $-133 a$ were proposed for the detection of more aggressive GI neuroendocrine neoplasms, and ISH was suggested as being useful in assisting qPCR findings [67]. $M i R-133 a$ downregulation in metastases compared to SBNET primaries was reported in another SBNET set [60], as well as its dysregulation in astrocytomas, hepatocellular carcinomas, or myocardial infarction. MiR-96 dysregulation has been associated with non-small cell lung cancer or breast cancer [67].

\section{Colonic/Rectal NET}

A miRNA profiling study [68] of what was named by the study investigators "colorectal NET" comprised a sample of 39 cases: carcinoids $(n=5)$, gland carcinoids $(n=11)$, adenocarcinomas $(n=9)$, small cell carcinomas $(n=8)$, and large cell neuroendocrine carcinomas $(n=6)$ (46\% male patients, and 25 controls with hemorrhoids, volvulus, or other benign, non-neoplastic diseases [60\% male]). The relationship between PTTG1 and MiR-186 was studied in tumor, blood, and stool. PTTG1 was found to be overexpressed, whereas $M i R-186$ was downregulated. MiR-186 was proposed as a regulator of PTTG1 expression and of infiltration/invasion in "colorectal NET."

A single case of colonic NET (an "atypical" - as described by the study authors - carcinoid, no histology provided) was mistakenly included in a series of colorectal cancers. The NET was sequenced and 38 upregulated miRNAs compared to normal mucosa were identified. These included MiR-7, -96, -1269, -1827, and -3177 [69].

In a separate study of 56 rectal carcinoids (no metastases in 77\%, no histology provided), MiR-885-5p (inhibitor of proliferation) was identified to be the most upregulated miRNA in tumors with lymphovascular invasion. Other upregulated miRNAs included MiR-135 and -486 [70].

\section{miRNAs in Blood}

\section{Source}

There is no clear source of circulating miRNAs (Fig. 2), although several hypotheses have been proposed. These include the following: (1) passive leakage of miRNAs from damaged cells (apoptosis, necrosis, chronic inflammation, tissue injury, or normal cells with a short half-life, e.g., platelets [71, 72]); (2) secretion within encapsulated microvesicles, exosomes, or shedding vesicles [34, 73, 74]; and (3) secretion through association with complexes that are RNA binding, such as Argonaute 2 (Ago2) [72, 75], nucleophosmin (NPM1) [76], or high-density lipoprotein [77]. At present, there is no consensus regarding the precise biological mechanisms underlying miRNA secretion [41].

\section{Function}

The biological function of circulating miRNAs is also unclear. It has been hypothesized that patterns of circulating miRNAs are specific to the secretory profile of a cell and its metabolic activity, and that circulating miRNA levels would therefore play a variety of roles in neoplasia
Malczewska/Kidd/Matar/Kos-Kudla/ Modlin 
[41]. Secreted miRNAs are thought to mediate cell-to-cell communication and gene regulation in an endocrine fashion $[78,79]$ based on the observation that miRNAs are selectively packaged for transport and secretion [76, 80]. Moreover, microvesicle membranes or protein carriers provide protection from RNases abundant in circulation, suggesting the "message" is protected [75]. Thirdly, circulating miRNAs can alter gene expression in recipient cells [74, 81-83].

\section{Measurement Techniques}

The advantages of measuring miRNAs include stability in biofluids and long-term storage. The major difficulty with miRNA assessment is the fact that there are significant inconsistencies in signatures from the same neoplasm [84]. A considerable amount of additional information about miRNA circulatory regulatory mechanisms and their putative biological function is prerequisite to defining their role as a clinically useful biomarker group.

\section{Sample Collection and Processing}

Standard procedures for sampling and processing are required to minimize miRNA variance (in type and expression) [85]. Furthermore, the correct compartment plasma or serum - should be determined. Although there is a correlation between serum and plasma miRNA levels [32], most studies indicate higher levels in plasma [86]. This may, however, reflect contamination by plateletderived miRNAs. One important step, which is unfortunately not taken as a standard, is ultracentrifugation to eliminate platelets prior to plasma analysis [41, 87]. Enrichment of miRNAs is not required, as the biofluids are enriched with small RNA species ( $>80 \%$ of the small RNA fraction, e.g., in serum) [32].

\section{miRNA Isolation}

Kits are preferred over manual protocols due to reproducibility issues. RNA isolation from biofluids is technically difficult because of circulating RNases and the high levels of protein in plasma. Quality control metrics are essential but are not yet routinely applied [88]. These can include the use of RNA spike-in (nonhuman oligo RNA) to monitor miRNA recovery. Hemolysis has a significant negative impact on miRNA signatures. The introduction of erythrocyte-derived miRNAs into serum or plasma may increase the concentration of some miRNAs up to 50 -fold [89]. As significant is the observation that intrinsic factors including age, gender, race, body mass index, diet, medication profile, vitamin supplementation, smok- ing, food intake, and altitude all affect circulating miRNA levels $[90,91]$. Age and gender matching and the use of fasting blood samples therefore are all a sine qua non [90].

\section{miRNA Detection}

Features that are problematic for accurate detection (quantification) include the short length of $\sim 22$ nucleotides (mature miRNAs) and the absence of a poly(A) tail, which precludes traditional reverse transcription or onestep PCR. In addition, mature miRNAs comprise $<0.01 \%$ of all RNAs. Further difficulties include distinguishing miRNAs that differ by only one nucleotide, identifying isomiRNAs, etc. Current miRNA profiling technologies include quantitative reverse transcription (PCR; one of the major approaches), microarray (including nCounter hybridization-based technology, not an array), and RNA sequencing (high-throughput next-generation sequencing, smaller-scale next-generation platforms, or singlemolecule sequencing technologies [88]).

\section{Data Analysis}

Accuracy is highly dependent on appropriate data normalization. Unfortunately, no definitive housekeeping miRNAs have been established. Small nucleolar RNAs such as RNU6B, RNU43, and RNU48, used as reference genes in intracellular miRNA-based studies, are not applicable to normalization of circulating miRNAs due to their degradation in biofluids which produces low or unstable values [92]. The most commonly utilized strategies for data normalization include global normalization, normalization to selected reference miRNAs, a synthetic spike-in, or some combination thereof $[93,94]$.

\section{miRNAs as a Circulating Marker in GEP-NETs}

Few studies have evaluated miRNAs in serum or plasma of GEP-NETs. They are all retrospective, and in only one instance [95] age- and gender-matched controls were utilized. Correlations with tumor tissue expression were established in a few cases. Overall, however, the quality of information derived was unsatisfactory. The data are included in Table 1.

\section{Gastric NET}

Upregulation of the X-linked MiR-222 (regulates $P 27^{K I P 1}$ ) was reported in the serum of hypergastrinemic patients with autoimmune atrophic gastritis and type 1 gastric NETs (in parallel with upregulation of $M i R-222$ in the gastric corpus mucosa) [50]. Treatment with the 
$\mathrm{CCK}_{2} \mathrm{R}$ antagonist netazepide (YF476), which inhibits NET secretion and proliferation, decreased the levels $(n=8)$ to values similar to those in healthy controls $(n=$ 10) $[50]$.

\section{Pancreatic NET}

Concordant upregulation of $M i R-193 b$ was noted in serum and tumor tissue (from predominantly well-differentiated tumors with a low Ki-67; cohort of serum donors without clinicopathological details) [53]. The miRNA expression levels in serum $(n=27)$ were similar to those in healthy volunteers (gender and age distribution not provided); however, $M i R-193 b$ was confirmed to be overexpressed [53]. In a separate study analyzing miRNAs capable of discriminating pancreatic cancer from healthy controls and other diseases, overexpression of MiR-1290 differentiated PDAC $(n=41)$ from PNET ( $n=18$; all well differentiated, $75 \%$ at stage I; mean patient age $\sim 57$ years, $61 \%$ male) with an AUC of 0.80 (95\% CI: 0.67-0.93). Other miRNAs significantly downregulated in PNETs compared to PDACs were MiR-584, -1285, -550a-5p, and -1825 [96]. It is unclear whether there were differences in expression of MiR-1290 between PNETs and controls ( $n=29$; mean patient age $\sim 43$ years, $86 \%$ male) [96]. There is evidence that $M i R-21$ is overexpressed in PNET plasma as opposed to that of chronic pancreatitis [47], but no data were provided in respect to the type and grade of the PNETs or whether MiR-21 was elevated compared to age- and gender-matched healthy controls.

\section{Small Intestinal/Bowel NET}

Alterations in miRNAs have been identified in blood, and their level may be affected by somatostatin analog (SSA) usage [97]. The serum expression of 9 miRNAs previously identified in tumor tissue [58] was studied in 48 SBNETs and 10 healthy controls. In 21 SSA-untreated SBNETS (7 primary tumors, 7 lymph node metastases, and 7 liver metastases; $20 \mathrm{G} 1$ and $1 \mathrm{G} 2$ ), the levels of $M i R$ $31,-129-5 p,-133 a$, and -215 were significantly lower than in the controls. In 21 SSA-treated SBNETs (7 primary tumors, 7 lymph node metastases, and 7 liver metastases; 17 G1 and 4 G2 - all with liver metastases) compared to 7 controls, the levels of MiR-96, -182, $-183,-196 a$, and $-200 a$ were significantly higher. Finally, when the panel of 9 miRNAs was evaluated in SSA-treated versus SSA-untreated SBNETs, the levels of MiR-96, -182, -183, -196a, and $-200 a$ were higher in the SSA-treated patients. For those miRNAs, SSAs were regarded as their regulators; however, no mechanism clarifying this epiphenomenon was proposed [58]. MiR-31, -129-5p, -133a, and -215 were decreased irrespective of SSA treatment. MiR-200a was upregulated only in the liver metastases, irrespective of SSA treatment, suggesting involvement in the metastatic cascade related to the epithelial-to-mesenchymal transition pathway [97].

In a separate study, 19 SBNETs ( 5 primary and 14 metastatic; mean patient age $\sim 60$ years, $63 \%$ female; no histological details provided) were compared with tissue from 19 age- and gender-matched controls [95]. This investigation identified 31 candidate miRNAs in plasma, which were then evaluated in 40 additional cases (mean patient age $\sim 66$ years, $55 \%$ female; $95 \%$ well differentiated) and controls. Four of the miRNAs were differentially expressed: $M i R-21-5 p$ and $-22-3 p$ were upregulated and MiR-29b-3p and -150-5p were decreased. These 4 miRNAs were then evaluated in a further cohort of 120 cases (mean patient age $\sim 69$ years, $49 \%$ female; $95 \%$ well differentiated; predominantly treated with SSAs, $72 \%$; treatment naïve, $23 \%$ ) and controls. Nineteen samples were removed from analysis due to hemolysis. The second validation confirmed MiR-21-5p and -22-3p to be elevated, with low levels of MiR-150-5p as a characteristic of metastasized SBNET. The AUC of these 3 miRNAs was 0.703. These miRNAs were unaffected by SSA use and appeared to be potentially associated with overall survival [95]. Specifically, low plasma expression of MiR-21-5p and -22-3p (both involved in inflammation and fibrosis) and MiR-21 (a known oncogene) and high expression of MiR-150-5p (involved in hematopoiesis, linked to several cancers including gastric cancer and osteosarcoma, promoting proliferation, involved in BCL signaling, and a potential marker of sepsis) were significantly associated with prolonged overall survival (median survival 6.1 vs. 3.5 years) [95].

Serum expression of $M i R-7-5 p$, previously noted to be upregulated in tumor samples (21 primary tumors and 9 liver metastases vs. NSB tissue), was measured in $32 \mathrm{pa}$ tients (18 males; G1 $[n=28], \mathrm{G} 2[n=2]$, and G3 [ $n=2]$; UICC stage I/II $[n=2]$, stage III [ $n=8$ ], and stage IV $[n=22]$ ). Serum MiR-7-5p was overexpressed in SBNETs compared to 25 controls; no correlation with age, gender, T stage, or UICC stage was identified [60].

\section{Correlating Circulating and Tumor miRNA Expression Profiles}

The most common hypothesis considers that tumor tissue miRNAs are released into the circulation either through secretion or as a function of cell death and necrosis, and that circulating levels will recapitulate tissue lev-
12

Neuroendocrinology DOI: $10.1159 / 000487326$
Malczewska/Kidd/Matar/Kos-Kudla/ Modlin 


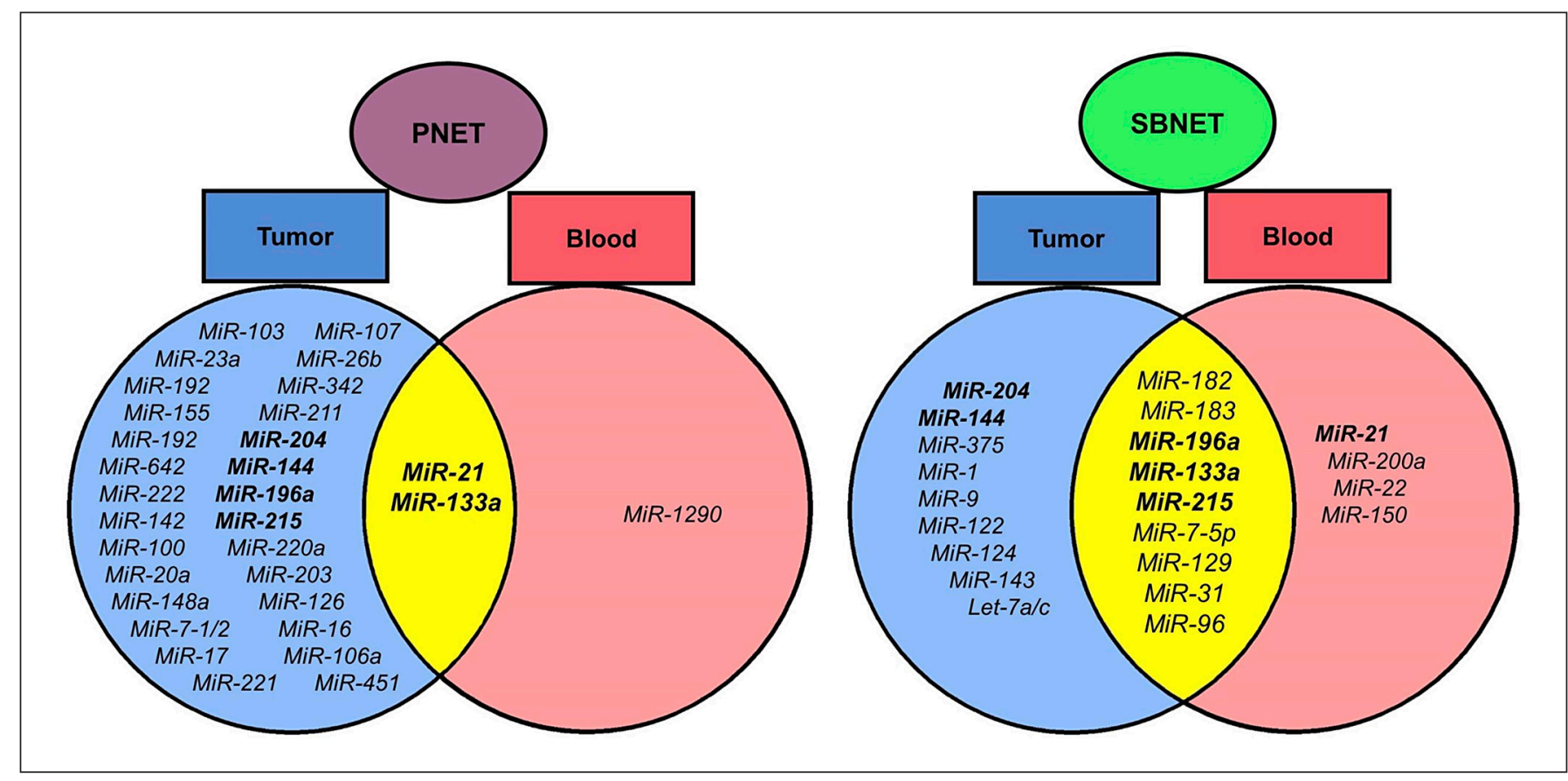

Fig. 4. Venn diagrams of dysregulated miRNAs in tumor and blood compartments of pancreatic (PNETs) and small bowel neuroendocrine tumors (SBNETs) showing aggregated data from published articles of studies which incorporated at least one internal stage of result validation $[47,51-54,56-58,60,61,63,65,67$, 95-97]. The yellow intersection areas of the Venn diagrams repre- sent miRNAs identified as being dysregulated in both the tumor and the blood compartment in either PNET or SBNET, irrespective of the direction of miRNA dysregulation. The miRNAs in bold represent miRNAs demonstrated to be dysregulated both in SBNET and in PNET. These miRNAs may be involved in NET development or, more broadly, in cancer genesis. els (i.e., function) as a "liquid biopsy." Selective miRNA secretion has been proposed, suggesting a role for miRNAs in cell-to-cell communication. The most plausible basis for a circulating miRNA signature in GEPNET reflects the dominant secretory phenotype of the neuroendocrine cell system [98]. Necrosis has been proposed for other cancers, but it is a relatively uncommon phenomenon in NETs. Hence, this mechanism is an unlikely source of extracellular miRNAs [53]. Alternatively, tumors may induce the secretion of particular miRNAs from other cells [53]. Abnormal miRNA profiles may also reflect nonspecific physiological responses to a growing neoplasm $[92,99]$. The latter may provide an explanation for any downregulation of circulating miRNAs.

The lack of concordance between circulating and tumor miRNA expression profiles in neoplasia is evident $[81,84,100,101]$. The correlation between circulating and tumor tissue miRNAs is poor, and substantial investigation is required to elucidate and define the relationship (Fig. 4). In particular, an assessment of the influence of preanalytical, analytical, or postanalytical variables that might confound the interpretation of circulating miRNA expression and its relationship to tumor-specific events is required. The detection and quantification of miRNAs remain challenging, since no adequate standardization or normalization strategy exists. Such endeavors have been initiated for PNETs and SBNETs by examining miRNAs from FFPE tissue samples ( 25 SBNETs and 10 PNETs). The goal was to establish the most stable/suitable small RNA for use as a tissue normalizer in real-time PCR experiments. One study identified SNORD61 and SNORD95 (SBNET) and SNORD95 and MiR-93 (PNET) as potential normalizers for miRNA expression, respectively [102]. These have, however, not been widely used.

\section{Current Status}

Given the complexity of the miRNA regulatory system, it seems unlikely that a single miRNA would be assigned a disease- or cancer-specific function [92]. This is 


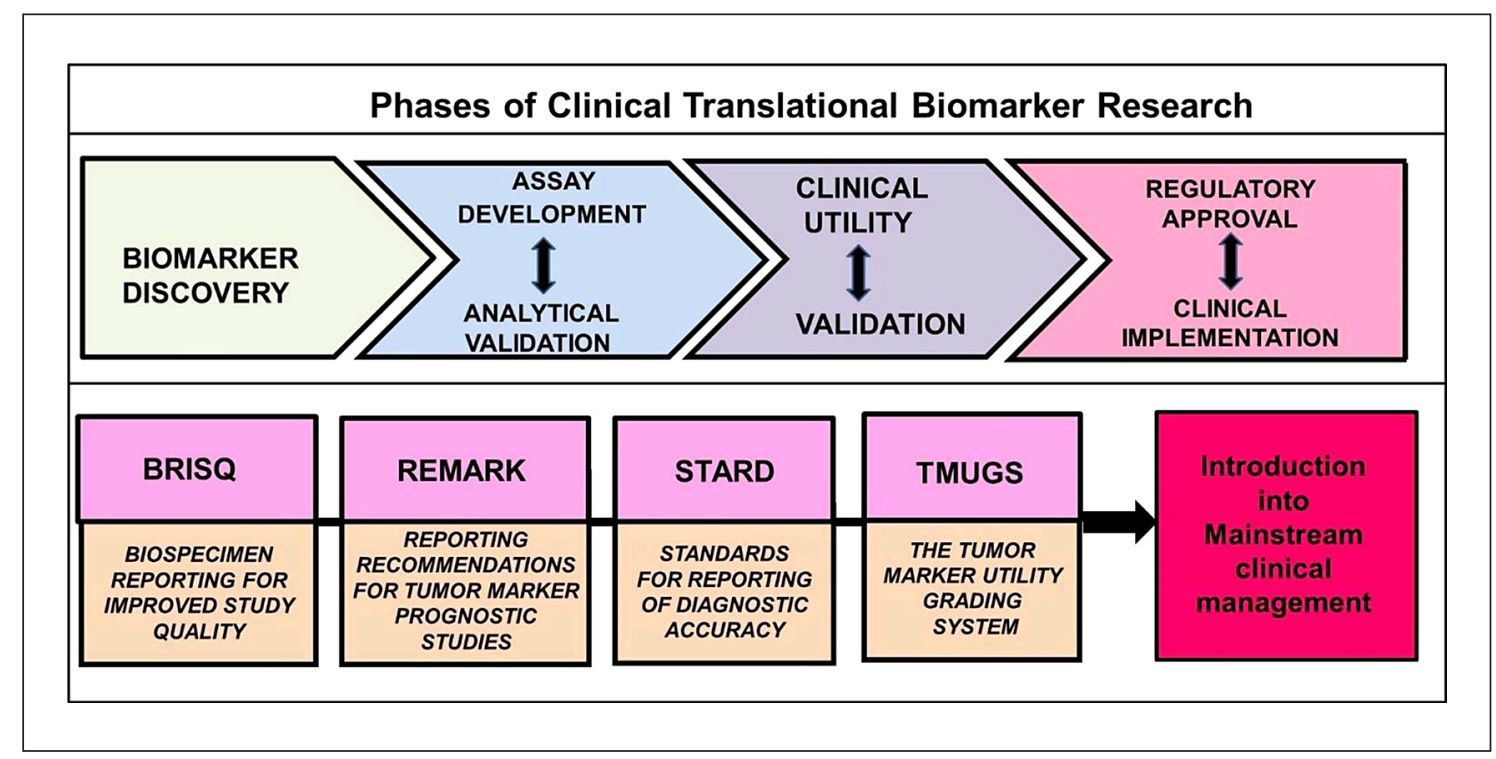

Fig. 5. Phases of clinical translational biomarker research. The initial investigation comprises a discovery phase, which includes an internal validation process. Candidate biomarkers (analytes) require two phases of validation: analytical validation (assesses how robust the test is in measurement of the analytes of interest) and clinical validation (demonstrates the clinical benefit of the assay). Following analytical and clinical validation, a biomarker assay enters a clinical implementation phase. This comprises four separate but interrelated elements, namely, regulatory approval, commer- cialization, health insurance coverage, and clinical guideline incorporation. Clinical translational biomarker research requires adherence to a series of guidelines, which include the Biospecimen Reporting for Improved Study Quality (BRISQ), Reporting Recommendations for Tumor Marker Prognostic Studies (REMARK), Standards for Reporting of Diagnostic Accuracy Studies (STARD), or Tumor Marker Utility Grading System (TMUGS), to ensure transparency and review of the study results. borne out by $M i R-21$, which is one of the most frequently reported of the circulating dysregulated miRNAs (cancers including: head and neck, breast, gastric, ovarian, pancreatic, prostate, colorectal, lung, neck, esophageal, liver, biliary tract, and nasopharyngeal, or diffuse large B-cell lymphoma, Hodgkin lymphoma, CNS lymphoma, glioblastoma, melanoma, or osteosarcoma) [3]. A more plausible scenario, consistent with the complexity of cancer biology, would be that multiple miRNA signatures would be required for tumor detection, prognosis, or prediction of treatment response [103].

Current approaches are limited by lack of concordance, data irreproducibility, and contradictory results of individual studies [84, 92, 99]. In particular, the most problematic issues reflect the scientific limitations of the detection strategies and the paucity of samples in validation sets that have not been adequately justified by power analysis $[84,92]$. Given the discordance between reported signatures as well as the differences between tumor and circulating levels (particularly downregulation in the blood), the introduction of optimization and standardization procedures is a critical requirement in miRNA studies [104]. To ensure the future utilization of miRNAs as a clinically useful biomarker in cancer management, miRNA-based research will need to comply with core established guidelines including the Biospecimen Reporting for Improved Study Quality (BRISQ) or Reporting Recommendations for Tumor Marker Prognostic Studies (REMARK) [105-107] and Standards for Reporting of Diagnostic Accuracy (STARD) [108]. The stages of development and clinical implementation of a biomarker incorporate analytical (technical) validation of the assay, clinical validation of the test (i.e., functions as a sensitive diagnostic), demonstration of a clinical benefit (i.e., aids clinical management decisions), and regulatory approval $[107,109]$ (Fig. 5). None of these have been rigorously established for miRNAs in NETs.

\section{Conclusions and Recommendations}

Despite numerous enthusiastic reports on miRNAs, their status as a biomarker still remains intangible (Table 2 ). This reflects a wide variety of limitations to the study
14
Neuroendocrinology DOI: $10.1159 / 000487326$
Malczewska/Kidd/Matar/Kos-Kudla/ Modlin 
Table 2. Assessment of miRNAs as clinical biomarkers

\begin{tabular}{|c|c|c|}
\hline Translational & $\begin{array}{l}\text { Tissue/circulating miRNA signatures specific to: } \\
\text { - cancer types at different stages } \\
\text { - other systemic diseases }\end{array}$ & $\begin{array}{l}\text { Lack of concordance in reported signatures (tissue vs. } \\
\text { circulating) for the same diseases and between the } \\
\text { studies }\end{array}$ \\
\hline Biological & $\begin{array}{l}\text { Involved in cancer genesis and pathogenesis of } \\
\text { diverse diseases, facilitating initiation of therapeutic } \\
\text { strategies }\end{array}$ & $\begin{array}{l}\text { Complexity of regulation and incomplete understanding } \\
\text { of overall biological function, currently limiting clinical } \\
\text { application }\end{array}$ \\
\hline $\begin{array}{l}\text { Clinical } \\
\text { accessibility }\end{array}$ & $\begin{array}{l}\text { Detectable in diverse biofluids (plasma, serum, saliva, } \\
\text { tears, urine, pleural fluid, peritoneal fluid, breast } \\
\text { milk, colostrum, bronchial lavage, amniotic fluid, } \\
\text { cerebrospinal fluid, seminal fluid) }\end{array}$ & $\begin{array}{l}\text { Methodological challenges in circulating miRNA } \\
\text { quantification, incomplete understanding of regulation } \\
\text { of miRNA release mechanisms }\end{array}$ \\
\hline Technical issues & $\begin{array}{l}\text { Significant stability in FFPE samples or biofluids; } \\
\text { resistance to harsh conditions and long-term storage }\end{array}$ & $\begin{array}{l}\text { Variations in quantification, no standardization in } \\
\text { preanalytical, analytical, and postanalytical strategies }\end{array}$ \\
\hline \multirow[t]{2}{*}{ Assay metrics } & $\begin{array}{l}\text { High AUC (sensitivity, specificity); prognostic and } \\
\text { predictive features of reported signatures }\end{array}$ & $\begin{array}{l}\text { Failure to meet accepted criteria proposed by (BRISQ, } \\
\text { REMARK, STARD, TMUGS) guidelines; numerous } \\
\text { inadequately powered biomarker-based studies with } \\
\text { protocols and data analysis not consistent with guideline } \\
\text { norms }\end{array}$ \\
\hline & $\begin{array}{l}\text { Prognostic and predictive features facilitating } \\
\text { individualized management }\end{array}$ & $\begin{array}{l}\text { Significant inter- and intraindividual variability of } \\
\text { miRNA expression profiles }\end{array}$ \\
\hline Theranostics & miRNA-based therapeutics in development & $\begin{array}{l}\text { Limitations in tissue-specific delivery of miRNA-based } \\
\text { therapeutics; stability in bloodstream; induced toxicity }\end{array}$ \\
\hline Current status & $\begin{array}{l}\text { Evolving field; considerable enthusiasm in both } \\
\text { clinical and scientific domains }\end{array}$ & $\begin{array}{l}\text { Currently minimal clinical applicability with substantial } \\
\text { scientific, methodological, and clinical utility concerns } \\
\text { to be resolved }\end{array}$ \\
\hline
\end{tabular}

FFPE, formalin-fixed, paraffin-embedded.

of the issue as to whether miRNA signatures in NET can be utilized. These include flaws in study design (e.g., poor age/gender matching), shortcomings of techniques, low inclusion numbers/inadequately powered groups, inclusion of heterogeneous cohorts, and the absence of independent validation sets. The modest information currently available suggests some potential biomarkers (e.g., $M i R-222$ [gastric] or MiR-21 [for the small bowel and perhaps the pancreas], and MiR-7-5p in SBNET) if the following criteria are used:

1. Concordant dysregulation of a candidate miRNA in the tumor and circulation, given the optimal biomarker would be detectable in both compartments (fulfilling the concept of a "liquid biopsy")
2. For blood-borne biomarkers, only upregulated miRNAs have been considered as suitable candidates (as discussed in Correlating Circulating and Tumor miRNA Expression Profiles)

3. Transparent design of miRNA biomarker-based studies, with at least one (internal) result validation

4. Proposed or already established biological role of dysregulated miRNAs in neuroendocrine tumorigenesis pathways

5. No evidence that the levels of proposed miRNAs may be affected by the treatment itself (not the treatment outcome) (e.g., SSAs)

However, given the complexity of cancer biology and the miRNA regulatory system, it seems unlikely that a single miRNA would be assigned a disease- or cancer- 
specific function, but rather that multiple miRNA signatures would be required. This will be the domain of future studies. At this time, there is no adequate evidence to support the proposal that measurement of circulating miRNA expression levels be considered pertinent to effectively guiding clinical management decisions.

A key unmet need in the consideration of neuroendocrine system-related miRNAs as biomarkers is the delineation of clusters of miRNA that define the diverse as- pects of the biology of NETs. Only once such information is available can consideration be given to the design of adequately powered studies to assess clinical utility. Whilst the use of miRNAs in NETs remains an attractive and au courant goal, understanding their biological relevance and resolving the current challenges and shortcomings in their study remain cardinal prerequisites before their clinical utilization.

\section{References}

$\nabla_{1}$ Siravegna G, et al: Integrating liquid biopsies into the management of cancer. Nat Rev Clin Oncol 2017;14:531-548.

$\checkmark 2$ Kulasingam V, Diamandis EP: Strategies for discovering novel cancer biomarkers through utilization of emerging technologies. Nat Clin Pract Oncol 2008;5:588-599.

3 Larrea E, et al: New concepts in cancer biomarkers: circulating miRNAs in liquid biopsies. Int J Mol Sci 2016;17:E627.

4 Modlin IM, et al: Neuroendocrine tumor biomarkers: current status and perspectives. Neuroendocrinology 2014;100:265-277.

5 Garzon R, et al: MicroRNA expression and function in cancer. Trends Mol Med 2006;12: 580-587.

6 Wu W, et al: MicroRNA and cancer: current status and prospective. Int J Cancer 2007;120: 953-960.

7 Lu J, et al: MicroRNA expression profiles classify human cancers. Nature 2005;435:834838.

8 Croce CM: Causes and consequences of microRNA dysregulation in cancer. Nat Rev Genet 2009;10:704-714.

9 Yang H, et al: Pathway analysis of cancer-associated microRNA targets. Int J Oncol 2012; 41:2213-2226.

10 Kosaka N, Iguchi H, Ochiya T: Circulating microRNA in body fluid: a new potential biomarker for cancer diagnosis and prognosis. Cancer Sci 2010;101:2087-2092.

11 Reid G, Kirschner MB, van Zandwijk N: Circulating microRNAs: association with disease and potential use as biomarkers. Crit Rev Oncol Hematol 2011;80:193-208.

12 Lindner $\mathrm{K}$, et al: Circulating microRNAs: emerging biomarkers for diagnosis and prognosis in patients with gastrointestinal cancers. Clin Sci (Lond) 2015;128:1-15.

13 Zhou L, et al: Circulating microRNAs in cancer: diagnostic and prognostic significance. Expert Rev Anticancer Ther 2012;12:283288.

14 Ma R, Jiang T, Kang X: Circulating microRNAs in cancer: origin, function and application. J Exp Clin Cancer Res 2012;31:38.
15 Allegra A, et al: Circulating microRNAs: new biomarkers in diagnosis, prognosis and treatment of cancer (review). Int J Oncol 2012;41: 1897-1912.

16 Khoury S, Tran N: Circulating microRNAs: potential biomarkers for common malignancies. Biomark Med 2015;9:131-151.

17 Bartel DP: MicroRNAs: genomics, biogenesis, mechanism, and function. Cell 2004;116: 281-297.

18 Lee RC, Feinbaum RL, Ambros V: The C. elegans heterochronic gene lin- 4 encodes small RNAs with antisense complementarity to lin14. Cell 1993;75:843-854.

19 Kozomara A, Griffiths-Jones S: miRBase: annotating high confidence microRNAs using deep sequencing data. Nucleic Acids Res 2014;42(database issue):D68-D73.

20 Valencia-Sanchez MA, et al: Control of translation and mRNA degradation by miRNAs and siRNAs. Genes Dev 2006;20:515-524.

-21 Hutvagner G, Zamore PD: A microRNA in a multiple-turnover RNAi enzyme complex. Science 2002;297:2056-2060.

22 Brodersen P, Voinnet O: Revisiting the principles of microRNA target recognition and mode of action. Nat Rev Mol Cell Biol 2009; 10:141-148.

23 Lee I, et al: New class of microRNA targets containing simultaneous $5^{\prime}$-UTR and $3^{\prime}$-UTR interaction sites. Genome Res 2009;19:11751183.

24 Rigoutsos I: New tricks for animal microRNAs: targeting of amino acid coding regions at conserved and nonconserved sites. Cancer Res 2009;69:3245-3248.

25 Friedman RC, et al: Most mammalian mRNAs are conserved targets of microRNAs. Genome Res 2009;19:92-105.

26 Lewis BP, Burge CB, Bartel DP: Conserved seed pairing, often flanked by adenosines, indicates that thousands of human genes are microRNA targets. Cell 2005;120:15-20.

27 Harfe BD: MicroRNAs in vertebrate development. Curr Opin Genet Dev 2005;15:410-415.

28 Lewis BP, et al: Prediction of mammalian microRNA targets. Cell 2003;115:787-798.

29 Ambros V: The functions of animal microRNAs. Nature 2004;431:350-355.
30 Chen B, et al: Roles of microRNA on cancer cell metabolism. J Transl Med 2012;10:228.

31 Avraham R, Yarden Y: Regulation of signalling by microRNAs. Biochem Soc Trans 2012; 40:26-30.

32 Chen X, et al: Characterization of microRNAs in serum: a novel class of biomarkers for diagnosis of cancer and other diseases. Cell Res 2008;18:997-1006

33 Lawrie CH, et al: Detection of elevated levels of tumour-associated microRNAs in serum of patients with diffuse large B-cell lymphoma. Br J Haematol 2008;141:672-675.

-34 Mitchell PS, et al: Circulating microRNAs as stable blood-based markers for cancer detection. Proc Natl Acad Sci USA 2008; 105: 10513-10518.

- 35 Basak I, et al: MicroRNAs as neuroregulators, biomarkers and therapeutic agents in neurodegenerative diseases. Cell Mol Life Sci 2016; 73:811-827.

36 Alipoor SD, et al: The roles of miRNAs as potential biomarkers in lung diseases. Eur J Pharmacol 2016;791:395-404.

37 Hashimoto N, Tanaka T: Role of miRNAs in the pathogenesis and susceptibility of diabetes mellitus. J Hum Genet 2017;62:141-150.

- 38 Paul P, et al: Interplay between miRNAs and human diseases. J Cell Physiol 2018;233: 2007-2018.

-39 Calin GA, Croce CM: MicroRNA signatures in human cancers. Nat Rev Cancer 2006;6: 857-866.

40 Cimmino A, et al: $m i R-15$ and $m i R-16$ induce apoptosis by targeting BCL2. Proc Natl Acad Sci USA 2005;102:13944-13949.

41 Ortiz-Quintero B: Cell-free microRNAs in blood and other body fluids, as cancer biomarkers. Cell Prolif 2016;49:281-303.

42 Kurozumi S, et al: Recent trends in microRNA research into breast cancer with particular focus on the associations between microRNAs and intrinsic subtypes. J Hum Genet 2017;62:15-24

43 Olson P, et al: MicroRNA dynamics in the stages of tumorigenesis correlate with hallmark capabilities of cancer. Genes Dev 2009; 23:2152-2165. 
44 Hiyoshi Y, Watanabe M: MicroRNAs in gastrointestinal cancer: a novel biomarker and its clinical application. J Cancer Metastasis Treat 2015;1:144-155.

45 Di Leva G, Croce CM: miRNA profiling of cancer. Curr Opin Genet Dev 2013;23:3-11.

46 Di Leva G, Garofalo M, Croce CM: MicroRNAs in cancer. Annu Rev Pathol 2014;9: 287-314.

47 Vicentini C, et al: Clinical application of microRNA testing in neuroendocrine tumors of the gastrointestinal tract. Molecules 2014;19: 2458-2468.

48 Ferracin M, et al: MicroRNA profiling for the identification of cancers with unknown primary tissue-of-origin. J Pathol 2011;225:4353.

49 Katada T, et al: MicroRNA expression profile in undifferentiated gastric cancer. Int $\mathrm{J}$ Oncol 2009;34:537-542.

50 Lloyd KA, et al: Gastrin-induced miR-222 promotes gastric tumor development by suppressing p2 $7^{\mathrm{kip} 1}$. Oncotarget 2016;7:4546245478.

51 Roldo C, et al: MicroRNA expression abnormalities in pancreatic endocrine and acinar tumors are associated with distinctive pathologic features and clinical behavior. J Clin Oncol 2006;24:4677-4684.

52 Jiang X, et al: miR-144/451 promote cell proliferation via targeting PTEN/AKT pathway in insulinomas. Endocrinology 2015; 156: 2429-2439.

53 Thorns C, et al: Global microRNA profiling of pancreatic neuroendocrine neoplasias. Anticancer Res 2014;34:2249-2254.

-54 Zhou HQ, et al: Integrative microRNAmRNA and protein-protein interaction analysis in pancreatic neuroendocrine tumors. Eur Rev Med Pharmacol Sci 2016;20:28422852.

55 Park M, et al: Characterization of gene expression and activated signaling pathways in solid-pseudopapillary neoplasm of pancreas. Mod Pathol 2014;27:580-593.

56 Lee YS, et al: High expression of microRNA196a indicates poor prognosis in resected pancreatic neuroendocrine tumor. Medicine (Baltimore) 2015;94:e2224.

57 Sadanandam A, et al: A cross-species analysis in pancreatic neuroendocrine tumors reveals molecular subtypes with distinctive clinical, metastatic, developmental, and metabolic characteristics. Cancer Discov 2015;5:12961313.

$58 \mathrm{Li} \mathrm{SC}$, et al: Global microRNA profiling of well-differentiated small intestinal neuroendocrine tumors. Mod Pathol 2013;26:685696.

59 Li SC, et al: Roles of miR-196a on gene regulation of neuroendocrine tumor cells. Mol Cell Endocrinol 2015;412:131-139.

60 Miller HC, et al: MicroRNAs associated with small bowel neuroendocrine tumours and their metastases. Endocr Relat Cancer 2016; 23:711-726.
61 Ruebel K, et al: MicroRNA expression in ileal carcinoid tumors: downregulation of $\mathrm{mi}-$ croRNA-133a with tumor progression. Mod Pathol 2010;23:367-375.

62 Shi Y, et al: miR-7-5p suppresses cell proliferation and induces apoptosis of breast cancer cells mainly by targeting REG $\gamma$. Cancer Lett 2015;358:27-36.

63 Heverhagen AE, et al: Overexpression of microRNA miR-7-5p is a potential biomarker in neuroendocrine neoplasia of the small intestine. Neuroendocrinology 2017, Epub ahead of print.

64 Yoshimoto T, et al: Pulmonary carcinoids and low-grade gastrointestinal neuroendocrine tumors show common microRNA expression profiles, different from adenocarcinomas and small cell carcinomas. Neuroendocrinology 2018;106:47-57.

65 Døssing KB, et al: Down-regulation of miR$129-5 p$ and the let-7 family in neuroendocrine tumors and metastases leads to up-regulation of their targets Egr1, G3bp1, Hmga2 and Bach1. Genes (Basel) 2014;6:1-21.

66 Nieser M, et al: Loss of chromosome 18 in neuroendocrine tumors of the small intestine: the enigma remains. Neuroendocrinology 2017;104:302-312.

67 Mandal R, et al: Analysis of miR-96 and miR133a expression in gastrointestinal neuroendocrine neoplasms. Endocr Pathol 2017;28: 345-350.

68 Wang M, et al: Roles of miR-186 and PTTG1 in colorectal neuroendocrine tumors. Int J Clin Exp Med 2015;8:22149-22157.

69 Hamfjord J, et al: Differential expression of miRNAs in colorectal cancer: comparison of paired tumor tissue and adjacent normal mucosa using high-throughput sequencing. PLoS One 2012;7:e34150.

70 Mitsuhashi K, et al: Analysis of the molecular features of rectal carcinoid tumors to identify new biomarkers that predict biological malignancy. Oncotarget 2015;6:22114-22125.

71 Laterza OF, et al: Plasma microRNAs as sensitive and specific biomarkers of tissue injury. Clin Chem 2009;55:1977-1983.

72 Turchinovich A, et al: Characterization of extracellular circulating microRNA. Nucleic Acids Res 2011;39:7223-7233.

73 Hunter MP, et al: Detection of microRNA expression in human peripheral blood microvesicles. PLoS One 2008;3:e3694.

74 Valadi H, et al: Exosome-mediated transfer of mRNAs and microRNAs is a novel mechanism of genetic exchange between cells. Nat Cell Biol 2007;9:654-659.

75 Arroyo JD, et al: Argonaute2 complexes carry a population of circulating microRNAs independent of vesicles in human plasma. Proc Natl Acad Sci USA 2011;108:5003-5008.

76 Wang K, et al: Export of microRNAs and microRNA-protective protein by mammalian cells. Nucleic Acids Res 2010;38:7248-7259.
77 Vickers KC, et al: MicroRNAs are transported in plasma and delivered to recipient cells by high-density lipoproteins. Nat Cell Biol 2011; 13:423-433.

78 Cortez MA, et al: MicroRNAs in body fluids the mix of hormones and biomarkers. Nat Rev Clin Oncol 2011;8:467-477.

79 Turchinovich A, et al: Circulating miRNAs: cell-cell communication function? Front $\mathrm{Ge}$ net 2013;4:119.

80 Théry C: Exosomes: secreted vesicles and intercellular communications. F1000 Biol Rep 2011;3:15.

81 Mittelbrunn M, et al: Unidirectional transfer of microRNA-loaded exosomes from T cells to antigen-presenting cells. Nat Commun 2011;2:282.

-82 Skog J, et al: Glioblastoma microvesicles transport RNA and proteins that promote tumour growth and provide diagnostic biomarkers. Nat Cell Biol 2008;10:1470-1476.

83 Pegtel DM, et al: Functional delivery of viral miRNAs via exosomes. Proc Natl Acad Sci USA 2010;107:6328-6333.

84 Jarry J, et al: The validity of circulating microRNAs in oncology: five years of challenges and contradictions. Mol Oncol 2014;8:819829.

85 Tuck MK, et al: Standard operating procedures for serum and plasma collection: early detection research network consensus statement Standard Operating Procedure Integration Working Group. J Proteome Res 2009;8: 113-117.

86 Wang K, et al: Comparing the microRNA spectrum between serum and plasma. PLoS One 2012;7:e41561.

87 McDonald JS, et al: Analysis of circulating microRNA: preanalytical and analytical challenges. Clin Chem 2011;57:833-840.

88 Pritchard CC, Cheng HH, Tewari M: MicroRNA profiling: approaches and considerations. Nat Rev Genet 2012;13:358-369.

89 Pritchard CC, et al: Blood cell origin of circulating microRNAs: a cautionary note for cancer biomarker studies. Cancer Prev Res (Phila) 2012;5:492-497.

90 Marzi MJ, et al: Optimization and standardization of circulating microRNA detection for clinical application: the miR-test case. Clin Chem 2016;62:743-754.

-91 Becker N, Lockwood CM: Pre-analytical variables in miRNA analysis. Clin Biochem 2013; 46:861-868.

92 Singh R, et al: Circulating microRNAs in cancer: hope or hype? Cancer Lett 2016;381:113121

93 Kok MG, et al: Normalization panels for the reliable quantification of circulating microRNAs by RT-qPCR. FASEB J 2015;29:38533862.

94 Sourvinou IS, Markou A, Lianidou ES: Quantification of circulating miRNAs in plasma: effect of preanalytical and analytical parameters on their isolation and stability. J Mol Diagn 2013;15:827-834. 
-95 Bowden M, et al: Profiling of metastatic small intestine neuroendocrine tumors reveals characteristic miRNAs detectable in plasma. Oncotarget 2017;8:54331-54344.

$\$ 96$ Li A, et al: MicroRNA array analysis finds elevated serum miR-1290 accurately distinguishes patients with low-stage pancreatic cancer from healthy and disease controls. Clin Cancer Res 2013;19:3600-3610.

97 Li SC, et al: Somatostatin analogs treated small intestinal neuroendocrine tumor patients circulating microRNAs. PLoS One 2015;10:e0125553.

$\checkmark 98$ Vazquez-Martinez R, Gasman S: The regulated secretory pathway in neuroendocrine cells. Front Endocrinol (Lausanne) 2014;5:48.

$\checkmark 99$ Witwer KW: Circulating microRNA biomarker studies: pitfalls and potential solutions. Clin Chem 2015;61:56-63.
100 Lodes MJ, et al: Detection of cancer with serum miRNAs on an oligonucleotide microarray. PLoS One 2009;4:e6229.

101 Boeri M, et al: MicroRNA signatures in tissues and plasma predict development and prognosis of computed tomography detected lung cancer. Proc Natl Acad Sci USA 2011;108:3713-3718.

$>102$ Sperveslage J, et al: Establishment of robust controls for the normalization of miRNA expression in neuroendocrine tumors of the ileum and pancreas. Endocrine 2014;46: 226-230.

103 Gandellini P, Giovannetti E, Nicassio F: MicroRNAs in cancer management: big challenges for small molecules. Biomed Res Int 2015;2015:982156.
104 D’Angelo B, et al: MicroRNAs: a puzzling tool in cancer diagnostics and therapy. Anticancer Res 2016;36:5571-5575.

105 McShane LM, et al: Reporting Recommendations for Tumour Marker Prognostic Studies (REMARK). Br J Cancer 2005;93: 387-391.

106 Moore HM, et al: Biospecimen Reporting for Improved Study Quality (BRISQ). J Proteome Res 2011;10:3429-3438.

107 Henry NL, Hayes DF: Cancer biomarkers. Mol Oncol 2012;6:140-146.

108 Bossuyt PM, et al: The STARD statement for reporting studies of diagnostic accuracy: explanation and elaboration. Ann Intern Med 2003;138:W1-W12.

109 Duffy MJ, et al: Validation of new cancer biomarkers: a position statement from the European Group on Tumor Markers. Clin Chem 2015;61:809-820. 